\title{
Molecular dynamics simulations of model oil/water/surfactant systems
}

\author{
K. Esselink *, P.A.J. Hilbers, N.M. van Os, B. Smit, S. Karaborni \\ Koninklijke/Shell-Laboratorium Amsterdam, P.O. Box 38000, Badhulsweg 3, 1030 BN Amsterdam, The Netherlands \\ Received 6 January 1994; accepted 16 March 1994
}

\begin{abstract}
Molecular dynamics simulations have been performed on amphiphilic molecules, oil and water to investigate surfactant behavior in water-like and oil-like solvents. Using a simple model for water, oil and surfactant molecules on a powerful parallel computer, we were able to simulate the adsorption of surfactants at the water/oil interface and the self-assembly of surfactant molecules into micelles. Simulations on various classes of surfactant molecules with different headgroup sizes and interactions show a strong dependence of the dynamics and morphology of surfactant aggregates on the surfactant structure. In the presence of an oil droplet, micelles induce the transfer of oil molecules from the oil droplet to the micelles by means of three mechanisms.
\end{abstract}

Keywords: Model; Molecular dynamics; Oil/water/surfactant systems; Simulations

\section{Introduction}

The application of computer simulations in studying complex physical systems on the molecular level has seen a dramatic increase over the last 10 years. The increase in computational power, and the accessibility of powerful computers to a large number of research workers are partly responsible for the popularity of computational methods such as the molecular dynamics (MD) and Monte Carlo (MC) techniques [1]. The MD method is based on the numerical solution of Newton's equations of motion for a set of atoms or molecules, i.e. the sampling of equilibrium averages is tied to the natural time evolution of the interacting atoms. In the MC approach, configurations are generated from random numbers obeying rules that ensure that mean values taken

\footnotetext{
*Corresponding author.
}

over the sample correspond to correct ensemble averages. Statistical mechanics are then used to calculate thermodynamic, transport and structural properties by providing a direct route from the microscopic to the macroscopic world. In MD simulations, static as well as dynamic properties can be calculated, while in MC simulations, only static properties are determined.

In our laboratory, molecular dynamics and MC simulations are being used as integral parts of mostly fundamental research, and steadily move to the domain of applied research. In particular, the MD technique is being used to study water/oil mixtures in the presence of surfactants. Surfactants in solution usually form aggregates such as micelles and vesicles, and adsorb at interfaces to form monolayers and bilayers. In the presence of two immiscible liquids, surfactants can induce the formation of microemulsions and macroemulsions $[2,3]$. To a large extent, the dynamics and the 
morphology of surfactant aggregates as well as the mechanisms of oil solubilization remain a hot subject of debate, due to the difficulty of using experimental techniques and the interpretation of the results. In this respect, simulations can help in the interpretation of experimental results and provide insight into processes at the molecular level.

This paper is structured as follows. In Section 2, we explain details of the models used to mimic water, oil and surfactant molecules. In the next section, some of the simulation details, such as parallel implementation and other computational aspects, are presented. In Section 4, results are presented on the adsorption of surfactants at an oil/water interface and the self-assembly of surfactants into micelles. In Section 5, the dynamics and morphologies of various surfactant structures are discussed and compared to theoretical predictions. In Section 6, we present the results of oil solubilization in micellar solutions.

\section{The model}

Computer simulations using the molecular dynamics technique are limited to studying several nanosecond periods. We are interested in processes such as surfactants leaving or entering a micelle (estimated at $10^{-9}-10^{-2} \mathrm{~s}$ ), and the break-up of a micelle $\left(10^{-2}-1 \mathrm{~s}\right)$ [4]. It is obvious that with a realistic model these phenomena cannot be observed. Therefore, it is important to construct a simple and powerful model that captures the essential features of water and surfactant molecules held to be responsible for the characteristic behavior of these systems.

Surfactants, water and oil molecules are represented using the simple model of Telo de Gama and Gubbins [5], and Smit et al. [6]. In this model, water molecules are treated as particles that interact via a Lennard-Jones potential $\Phi$ with a cut-off large enough to include excluded volume effects and attractive forces $\left(R_{\mathrm{c}}=2.5 \sigma\right.$, where $R_{\mathrm{c}}$ is the cut-off distance and $\sigma$ is the size parameter in the Lennard-Jones potential). Oil-oil interactions are treated similarly to water-water interactions, while oil-water interactions are modeled using only the repulsive part (excluded volume) of the
Lennard-Jones potential $\left(\boldsymbol{R}_{\mathrm{c}}=1.12 \sigma\right)$. The LennardJones potential is shifted to zero at the cut-off, resulting in a continuous potential:

$$
\Phi(r)= \begin{cases}4 \epsilon\left[\left(\frac{\sigma}{r}\right)^{12}-\left(\frac{\sigma}{r}\right)^{6}\right]+E_{\text {shift }} & \text { if } r<R_{\mathrm{c}} \\ 0 & \text { otherwise }\end{cases}
$$

where $r$ is the distance between the two interacting particles. The energy shift $E_{\text {shift }}$ thus depends on the cut-off used. We have assumed that $\sigma=1$ and $\epsilon=1$ for all interactions.

Neighboring particles on the same molecule are connected by harmonic springs of length $\sigma$, the size parameter in the Lennard-Jones potential:

$$
U(r)=\frac{1}{2} k(r-\sigma)^{2}
$$

The value of $k$ is chosen such that at any time $98 \%$ of the connected atoms are within $2 \%$ of the average value $\sigma$.

Note that in this model, we did not take hydrogen bonds into account explicitly, nor did we model ionic interactions. Similarly, the intramolecular interactions did not include any torsion or bending potentials.

The different molecules used in the simulations described here are water molecules made of one water-like particle, oil molecules made of one oillike particle, oil molecules made of linear chains of two (T2), three (T3) and five (T5) oil-like particles, and surfactant molecules made of a linear chain of four oil-like and four water-like particles (H4T4) or five oil-like particles and a head group that consists of two water-like particles (H2T5), or two or three water-like particles centered around another water-like particle (H2HT5 or H3HT5). In Fig. 1, a schematic drawing of these molecules is given.

\section{Computational aspects}

All simulations were performed at constant temperature and volume. Periodic boundary conditions were imposed in all three dimensions. The 


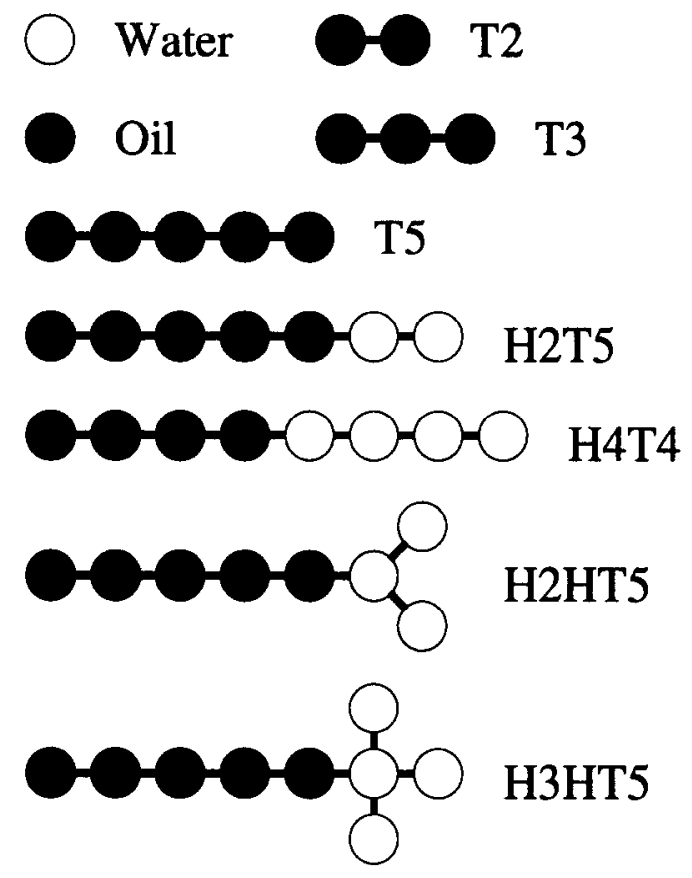

Fig. 1. Schematic drawing of the model surfactant, water and oil molecules.

particle density $\rho$ was $0.7 \sigma^{-3}$ in systems with 39304 or 32000 particles. The equations of motion were solved using Verlet's integration scheme [7]. Using a time step of $\delta t=0.005 \tau_{0}$ (where $\tau_{0}=\sigma(m / \epsilon)^{1 / 2}$ ), energy conservation was guaranteed.

The micelles in our simulations are not modeled explicitly. They occur as a spontaneous grouping of a certain set of surfactants. Therefore, we need a special algorithm to identify the different micelles, given a universe with water, oil and surfactants. In our definition, two surfactants belong to the same micelle if the distance between the two tails is at most $1.5 \sigma$. This distance is measured by taking the minimum of the distances between any two particles of the tails. The different micelles are then found using a standard connected component algorithm [8].

Because of the use of relatively large systems, we used parallel computers for the simulations. The molecular dynamics technique is well suited for parallel computations, since the necessary computations are the same for all particles. Two phases can be distinguished: in the first phase, the forces on each particle are determined, and in the second phase, the displacements of the particles are determined from the forces. Macroscopic properties of the system can also be determined at this stage. The latter phase is especially trivial to parallelize. The first phase is usually more difficult to parallelize since the processors need to cooperate (exchange information) in order to compute the forces on the particles.

The two main techniques for exploiting parallelism are particle parallelism and geometric parallelism. Using the first technique, particles are assigned to processors $[9,10]$. Each processor continually calculates forces and the new positions for its own particles. The initial distribution of particles remains unchanged during the simulation and can be chosen such that the workload is evenly distributed among the processors. However, despite the short-range nature of the potential, it is necessary for each processor to communicate with all others to determine whether any two particles interact. The communication costs will therefore increase with the size of the computing network, seriously degrading performance of large computer networks.

When the geometric parallelism is applied, space, not particles, is assigned to processors [11,12]. During the computation, a processor calculates the trajectories of all particles it finds in its space. Because of the movement of the particles, some particles may enter a processor's space, others may leave. For this reason, processors continually need to redistribute the particles to make sure that each one has the right subset. The rclevant question is which part of the universe should be assigned to a processor. Here, the short-range nature of the Lennard-Jones potential can be used. Since the interaction does not extend over distances larger than $R_{\mathrm{c}}$, it is not necessary to exchange information over long distances. As in the "linked-list" method [1], we assume that the simulation box is divided into a number of cells, such that particles only interact with other particles in the same cell or in neighboring cells. Hence we can associate with a cell a search space of adjacent cells in which particles reside that have to be investigated for interaction. In Ref. [13] we have considered several choices for a cell like an octahedron, a rhombic dodecahedron, and a cube, to determine which 
shape leads to the smallest search space. The cube turns out to be the best, because it has an optimum volume and number of neighbors (see Fig. 2).

Having divided the simulation box into a regular cubic lattice of cells, and assuming a homogeneous distribution of particles, a good load balance can be achieved by assigning the same number of cells to all processors. In order to minimize communication costs, which depend on both the number of cells that have to be communicated, and the distance between the two communicating processors, cells have to be assigned judiciously to processors. If we have a square torus of processors, the most "natural" mapping (which is also mostly used) is the orthogonal projection of the universe onto the torus of processors. This mapping has the advantage that the wrap-around in the $z$ direction can be obtained without the need for extra communication. In Ref. [13] we have shown that for processor networks of up to size $32 \times 32$, this column mapping is the most efficient, but for larger networks it is better to map objects which are more spherical.

The use of parallel computers makes it possible to perform simulations at a scale at which the use of conventional supercomputers, such as vector machines, would be too expensive. A 400 processor Transputer network is approximately as fast as a single Cray YMP processor.

\section{Surfactants in a water and oil mixture}

One of the first simulations we performed was carried out on a system comprising a water and oil mixture, including a small percentage of H2T5 surfactants [14]. The simulations were performed at constant temperature $\left(T=1.0 \epsilon / k_{\mathrm{B}}\right.$, where $k_{\mathrm{B}}$ is Boltzmann's constant) and volume. The temper-
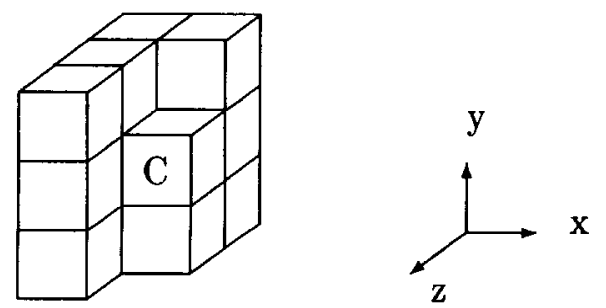

Fig. 2. One cell (C) with 13 neighbors (three-dimensional). ature was kept constant by scaling the velocities every 200 th time step. Periodic boundary conditions are imposed in all three directions.

Initially, a number of particles were placed on an f.c.c. lattice with size $30.4 \sigma \times 30.4 \sigma \times 60.8 \sigma$. The density obtained in this instance was $\rho=0.7 \sigma^{-3}$, with 39304 particles. Surfactants were introduced in the following way. First a randomly chosen particle on the f.c.c. lattice was connected with one of its randomly chosen neighbors. The latter was then connected again to one of its neighbors. This procedure was repeated until the desired number of surfactants with the desired length was formed. This initialization guarantees a spatially random distribution of surfactants. All the remaining particles in one half of the periodic box were water and in the other half, oil. The surfactant concentration ranged from 0.75 to $3 \%$. The system was equilibrated for at least 100000 time steps $(\delta t=$ $0.005 \tau_{0}$ ), followed by a production run of at least another 100000 time steps.

Fig. 3 shows density profiles for a surfactant concentration of $3 \%$. The surfactants are preferentially adsorbed at the interface as a monolayer. The segment distribution reflects the expected orientational ordering (hydrophilic heads towards water). The density profile of the water shows

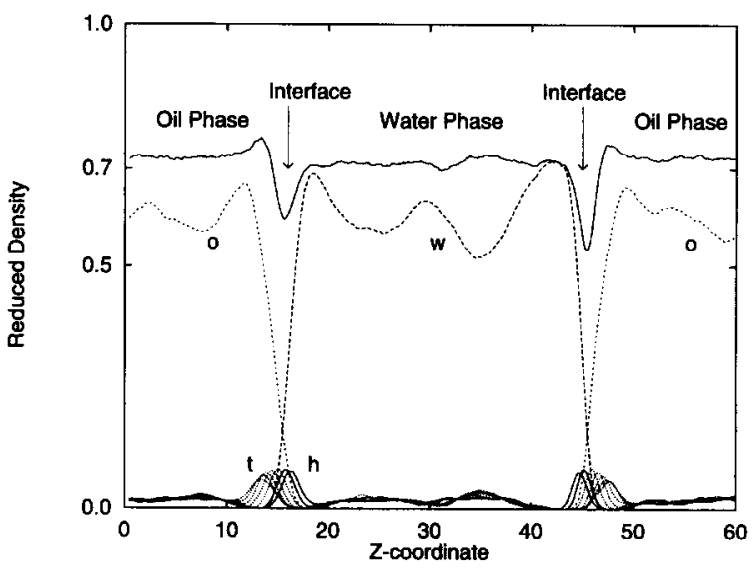

Fig. 3. Average density of water, oil and surfactants for a $3 \%$ H2T 5 surfactant concentration. The top solid curve gives the total density; the broken curves labeled o and $w$ represent the density of oil and water respectively; and the two solid curves labeled $h$ and the five broken curves labeled $t$ represent the head and tail groups. 
pronounced oscillations, due to the spontaneous formation of large micelles. The density profile of the oil phase also shows some oscillations but these are much less significant. A possible explanation of these observations is the following. We recall that the structure of simple liquids [15] and even of some more complex systems such as liquid crystals [16] is largely due to hard core interactions. If one imagines a micelle to be a hard sphere and the monolayer to be a wall, the system is equivalent to a hard-sphere fluid confined between two parallel plates. In such a system, packing constraints are known [17] to cause characteristic oscillations in the density profile. Similarly, packing constraints on the micelles cause oscillations in the micelles distribution. Since the total density in the water phase is constant (see Fig. 3) the water molecules will fill the remaining space and thus the water density will oscillate, with a period of the order of the diameter of the micelle. This explanation is also consistent with the phenomena observed in the drainage of thin foam films containing micelles. Measurements of the thin film width as a function of time show that the width decreases in steps of magnitude equal to the micellar diameter [18].

A typical example of the instantaneous arrangement of the surfactants at a $1.5 \%$ surfactant concentration is shown in Fig. 4. In the water phase, a micelle has formed spontaneously. Fig. 5 shows a projection of one of the monolayers on the $x, z$ plane and on the $x, y$ plane as seen from the water side. This figure shows only those surfactants which are part of the monolayer. They are not distributed homogeneously over the monolayer, but tend to cluster into small domains. This observation might have significant repercussions on the interpretation of adsorption experiments [19]. The clustering of surfactants in the monolayer can provide an alternative explanation to the non-diffusion-limited behavior of surfactant adsorption at a liquid/liquid interface. In other words, adsorption behavior is dependent on the dynamics of free monomers as well as on the dynamics of two-dimensional surfactant clusters.

We have performed some preliminary calculations of the interfacial tension (IFT) in the absence of micclles [20]. These calculations predict correctly the effect of chain length and chain branching

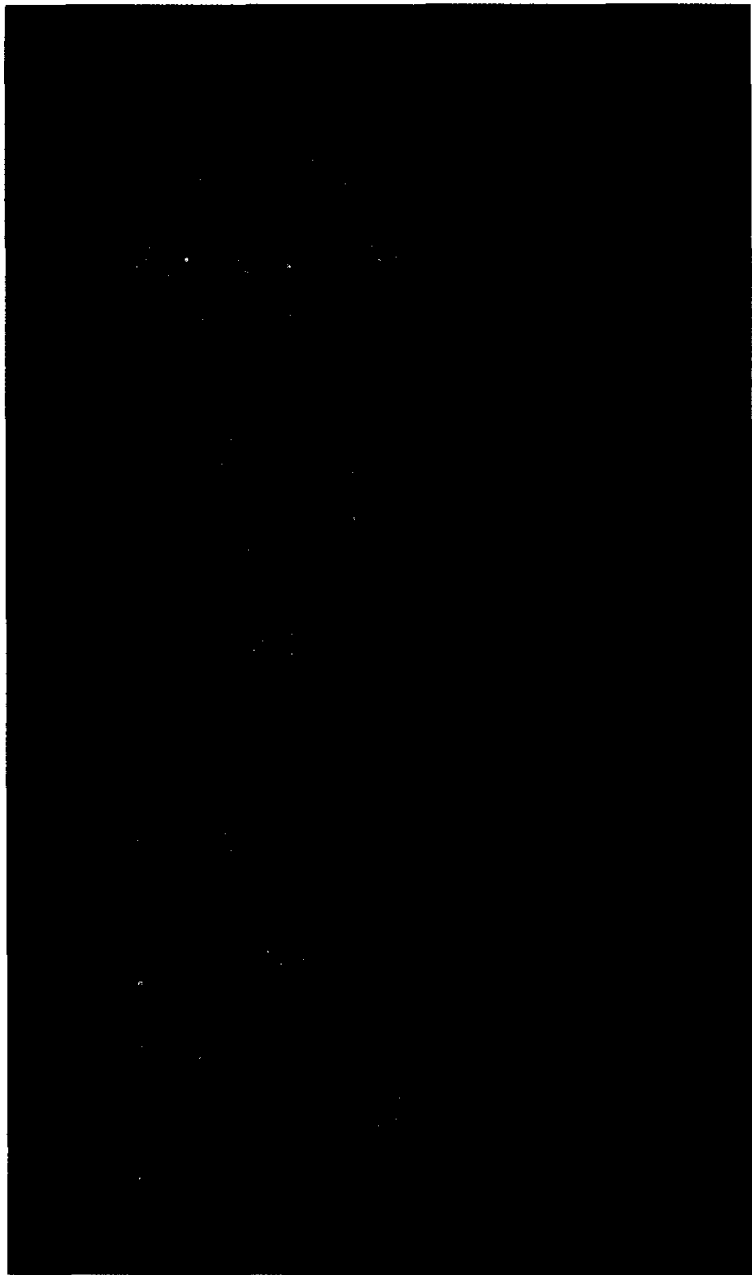

Fig. 4. Snapshot of H2T5 surfactants in water and oil (surfactant concentration, $3 \mathrm{~mol} \%$ ). Headgroups are displayed in yellow and tail segments are shown in red.

on the IFT, and compare quite reasonably with experimental data and self-consistent field lattice theory for adsorption and/or association (SCFA) results [21].

A novel aspect of the present work is that the interface as well as the micelles are described with one model, whereas other simulation work has been mainly concerned with either isolated micelles [22] or a monolayer [23]. It is therefore interesting to compare our simulations with experimental data on a real system of micelles near an interface. Recently, specular ncutron reflection expcriments were performed on the air/water interface of 

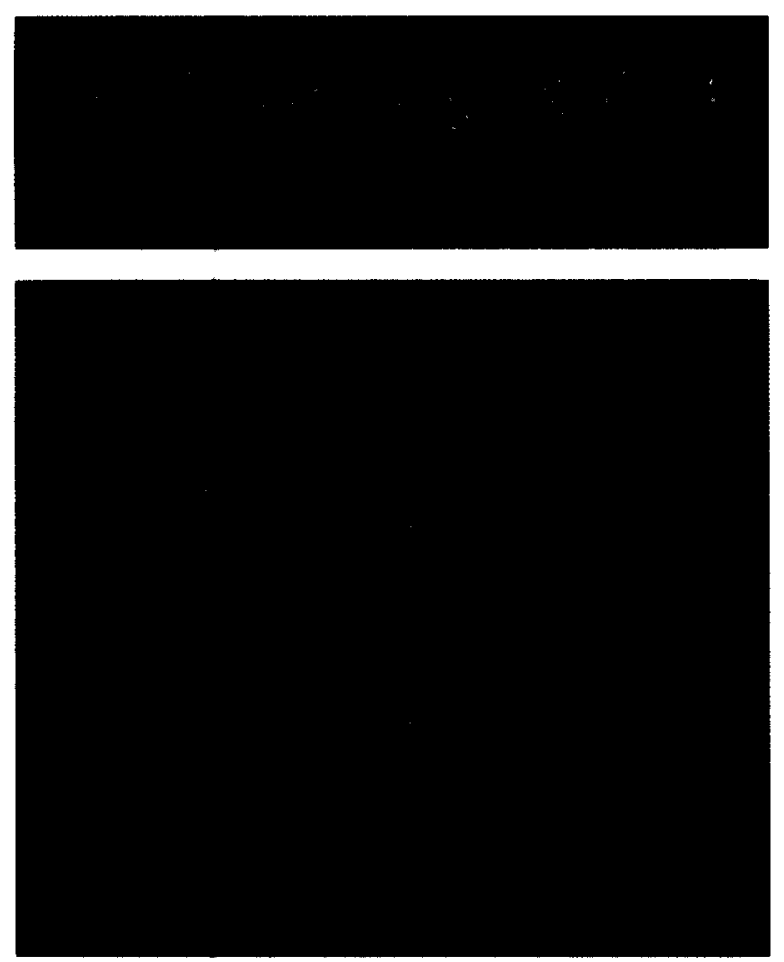

Fig. 5. Snapshot of a projection of one of the monolayers of H2T5 surfactants on the $x, z$ plane (top) and on the $x, y$ plane as seen from the water side (bottom). See Fig. 4 for the color scheme.

solutions containing ionic surfactants $[24,25]$ or non-ionic surfactants [25]. The experiments reveal an "unexpected" enhancement in the reflectivity of $\mathrm{D}_{2} \mathrm{O}$ once the surfactant concentration exceeds the critical micelle concentration. This enhancement has not yet been explained satisfactorily. It is therefore interesting to note that the oscillations of the density profile of water as observed in our simulations should give rise to just such an enhancement of the reflectivity. The location of the maximum, which is a measure of the period of the density oscillations, depends on the size of the micelle, which in turn depends on the type of surfactant. Indeed, such a dependency has been observed in the reflectivity experiments [25]. It would be interesting to test our hypothesis experimentally by adding deuterated oil to the solution. The oil molecules will dissolve in the interior of the micelles. This results in an oscillating density profile of the oil close to the interface. This should give rise to an enhancement of the reflectivity as well.

Lee et al. [25] have stated that an oscillating water density profile would explain the enhancement. They attribute these oscillations to a complicated layered structure of water and surfactant bilayers. Our simulations suggest that these oscillations can result from the packing of micelles near an interface. Recently, this observation has been confirmed by Lu et al. [26].

A common question with these kinds of simulations concerns the time frame, i.e. whether or not the system has reached equilibrium. We performed simulations of up to 1000000 time steps, and found that the surfactants in the water phase tend to keep clustering until one bilayer-like cluster is left. Our subsequent efforts dealt with a systematic study of the dependence of micelle morphology and dynamics on the structure of the surfactant used, as well as system properties like temperature. These are reported in the next section.

\section{Surfactants in water}

It has been known for quite some time that amphiphilic molecules, which contain a hydrophobic tail and a hydrophilic head, can form a variety of aggregates with properties different from those of the unassembled molecules. Indeed, spherical micelles, rod-like micelles, bilayers, reverse micelles, vesicles and even worm-like micelles have all been observed in the laboratory and this astonishing polymorphism forms the basis of many biochemical processes and is being applied in many industrial and household applications.

We can understand the shape of such aggregates by considering the role of molecular packing constraints. At the surface of the aggregate, where hydrophobic tail particles can come into contact with solvent water molecules, the interfacial tension will tend to decrease the average headgroup area of the amphiphile; this is counteracted by headgroup repulsion forces and chain packing constraints. This constant tug-of-war leads to an optimal headgroup area $A_{0}$ of the amphiphilic molecule at the surface of the aggregate. According to Israelachvili [27], simple geometrical considerations can then be applied to find the most favorable 
shape of the aggregate. If $V$ is the volume of the hydrocarbon tail of the amphiphile and $L$ is the maximum length of the tail, and $A_{0}$ equals the optimal surface area (the area at which the free energy is minimal), then spherical micelles will form if a quantity called the packing parameter $P=V /\left(A_{0} L\right)$ is less than $1 / 3$. For values of $P$ between $1 / 3$ and $1 / 2$, rod-like micelles will form, whereas bilayers will appear for $P$ values between $1 / 2$ and 1 . Finally, for $P$ larger than 1 , the preferred morphology is that of the reversed micelle. In other words, this geometric parameter tells us something about local curvature: small values of $P$ correspond to strongly curved micellar aggregates, large values of $P$ are indicative of large or inverted structures.

The relationship between $P$ and aggregate morphology holds for single-chain amphiphiles such as $n$-dodecyltrimethylammonium bromide (DTAB) for which $P=0.32$ and which forms spherical micelles in water [28]; when $\mathrm{KBr}$ is added, the headgroup charges will become screened, the electrostatic repulsion between the headgroups will diminish, and the resultant decrease of $A_{0}$ leads to $P=0.46$ and the formation of rod-like micelles [29]. When a second hydrocarbon chain is added to this headgroup to form di-n-dodecyldimethylammonium bromide (DDAB), the volume of the hydrophobic portion of the molecule doubles, $P$ becomes 0.84 and one observes the formation of lamellar phases in water [30]. Very similar conclusions were observed by Nusselder and Engberts [31] for the class of the 1,4-dialkylpyridinium halide amphiphiles.

If the geometry of an amphiphile determines the morphology of the aggregate, one wonders whether this can also be observed in simulations of spontaneous self-assembly in water.

\subsection{Surfactant with a small headgroup H2T5}

Our first attempt was to simulate a surfactant molecule that has a linear tail of five beads and a linear headgroup of two beads. In addition to the interactions mentioned in Section 2, we constrained the headgroups to interact via the full LennardJones potential; in other words, the headgroups are attractive. The simulation results for this surfactant show that spherical structures are formed initially, which collide to form disk-like structures.
At later stages, bilayers are formed via the collision between disks (see Fig. 6). The coalescence of two disks seemed to proceed in a very structured manner, in the sense that most collisions between disks were not successful. Only those collisions in which two disks encounter each other from the rim were successful (see Fig. 7). A calculation of the geometrical factor $P$ for this surfactant shows that it has a value of approximately 1 . For these values of $P$, one would expect bilayer structures in agreement with our simulation results.

\subsection{Surfactant with a chain-like headgroup H4T4}

The second surfactant that we have studied was a symmetric surfactant that has a linear tail of four oil-like beads and a linear headgroup of four waterlike beads. Similarly to H2T5, the interactions between the H4T4 headgroups were attractive. One would expect from the geometry of this surfac-
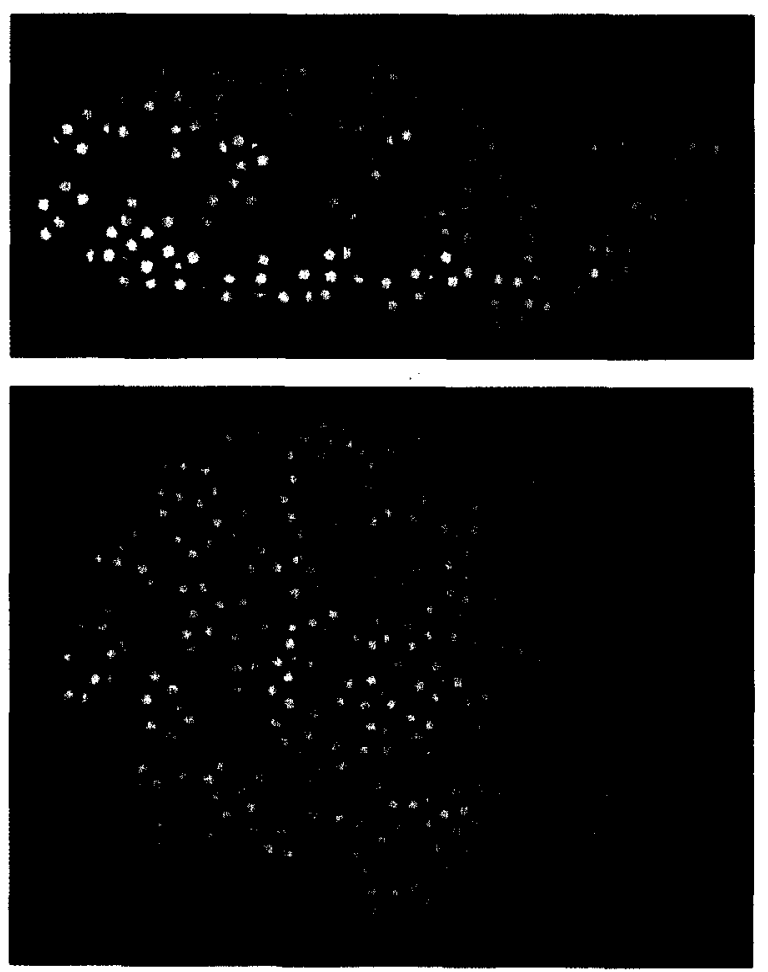

Fig. 6. Snapshot of the system of disk-like micelles of H2T5 surfactants, from the side (top) and from the top (bottom). See figure 4 for the color scheme. 


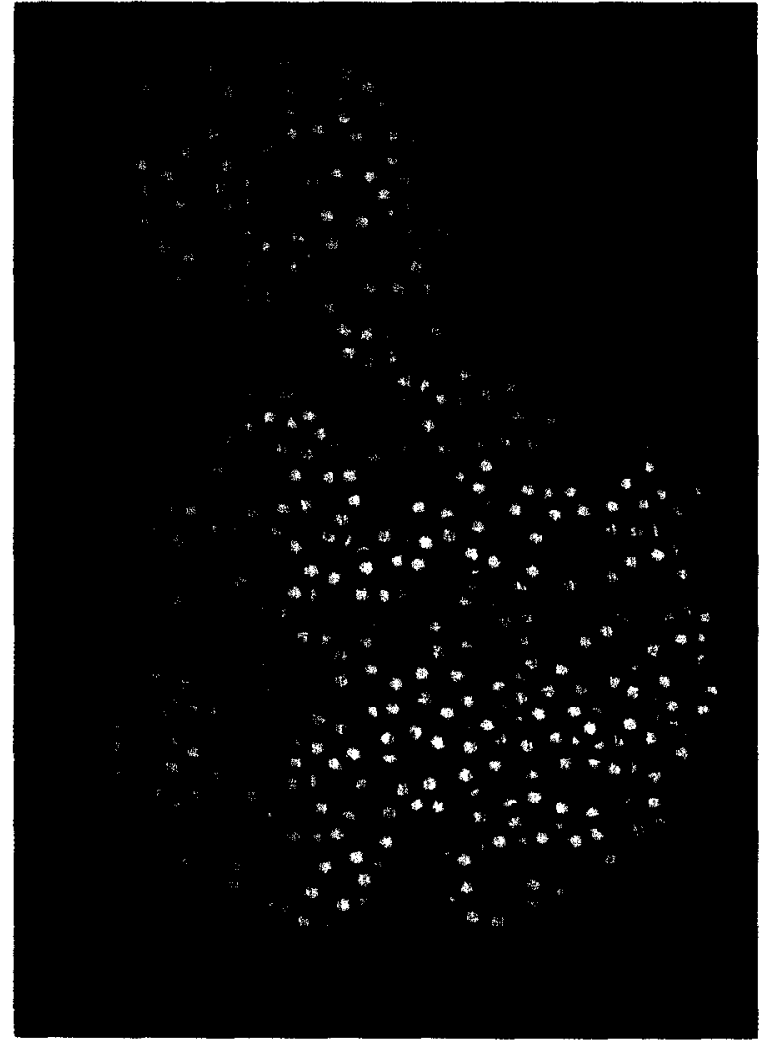

Fig. 7. Snapshot of the system of two colliding disk-like micelles of H2T5 surfactants, from the top. See Fig. 4 for the color scheme.

tant that aggregates with low curvature are formed. However, this was not the case. We see that the aggregates have spherical-like shapes (see Fig. 8) with a wide distribution in size ranging from 15 to 80. Snapshots of some of the aggregates show that the main reason for the formation of spherical shapes is the conformation of the headgroups at the micelle/water interface. It appears that only very few of these headgroups are truly extended linearly into the solvent, while most headgroups have assumed an area at the interface that is larger than that of the fully extended headgroup. To understand the behavior of these headgroups, one perhaps should think about the behavior of flexible molecules such as alkanes in a non-polar solvent of Lennard-Jones particles. Simulations of these systems [32] show that the alkanes are not fully stretched and have, at most, two-thirds of the

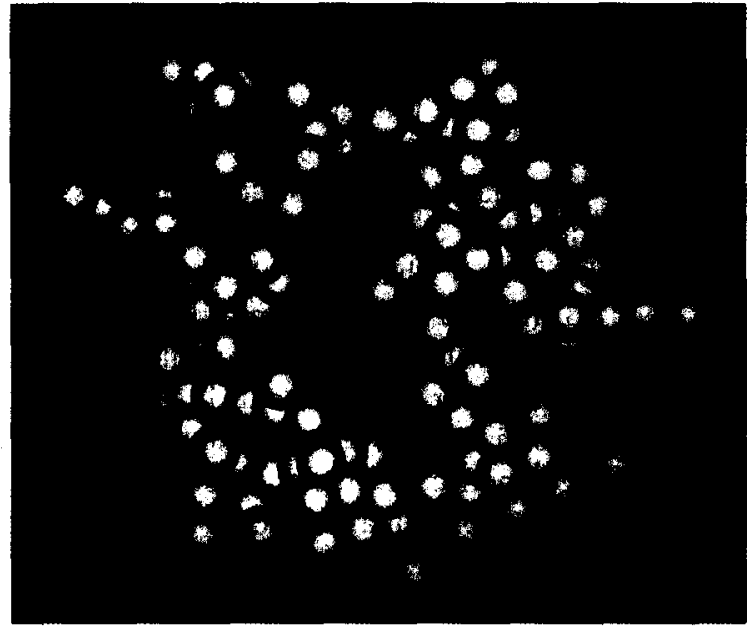

Fig. 8. Snapshot of a spherical micelle of H4T4 surfactants. See Fig. 4 for the color scheme.

dihedral angles in the trans conformation. One suspects that the aggregate behavior displayed by H4T4 would be also exhibited by semi-fluorinated, semi-hydrogenated molecules in a solvent of $n$ alkanes or fluorinated $n$-alkanes [33].

\subsection{Surfactant with a large headgroup H2HT5}

Following our simulations with linear surfactant molecules, we have studied the behavior of those molecules for which the headgroup is branched as shown in Fig. 1. Similarly to previous simulations, the interactions between the headgroups were attractive. Simulation results show that most aggregates have oblate-like shapes (see Fig. 9). The increase in shape curvature can be easily predicted for these types of surfactants by simply using a larger value of $A_{0}$ in the geometrical criteria discussed above.

\subsection{Surfactant with a bulky headgroup H3HT5}

As a subsequent step in increasing the bulkiness of the headgroup, we added one bead to the headgroup and made the interactions between the headgroups repulsive (Fig. 1). As would be expected from the geometrical criteria discussed above, shapes with a high curvature are formed. Fig. 10 shows some of these aggregates which have, 


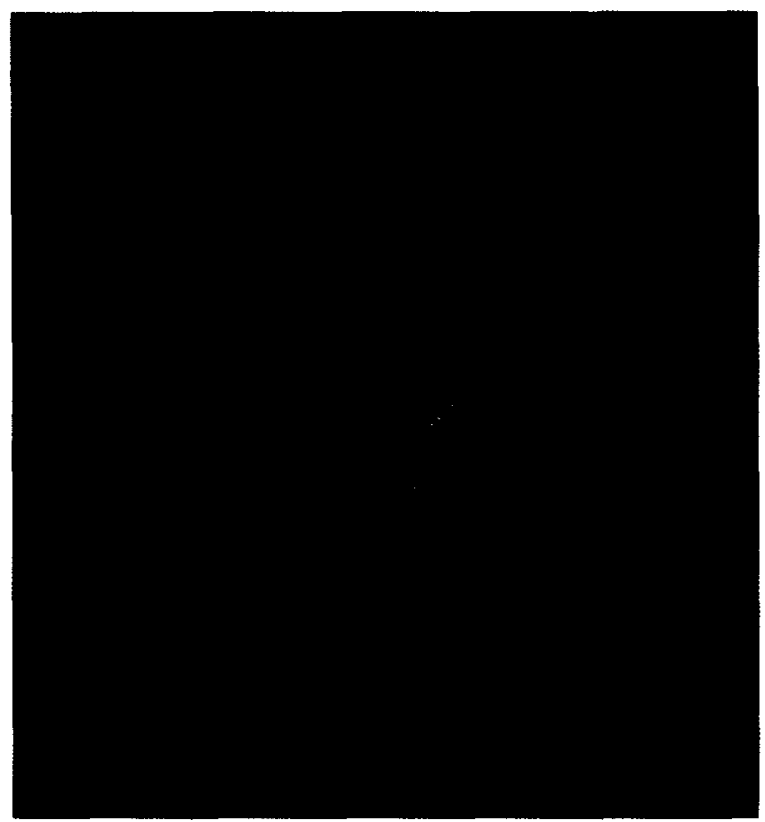

Fig. 9. Snapshot of the system of oblate-like micelles of H2HT5 surfactants. See Fig. 4 for the color scheme.

on average, spherical shapes, but show significant shape fluctuations. This simulation was followed for a long duration, after which we calculated the size distribution of the micelles (see Fig. 11). This size distribution is reminiscent of that commonly found for ionic surfactants with large headgroups such as octyl or nonyl sulfates. The average size of the micelles seems to be very strongly affected by a change in temperature as shown in Fig. 12. A $4 \%$ increase in absolute temperature induces a significant decrease of $15 \%$ in the average micellar size. This is not surprising considering that fluorescence quenching experiments predict that a $10 \%$ increase in temperature induces a decrease of $48 \%$ in the average aggregation number of sodium ortho-(1-decyl)benzenesulfonate at a concentration of $0.05 \mathrm{M}$ [34].

\section{Oil solubilization}

To study oil solubilization, a micellar solution was created by adding free monomeric surfactants to a box containing water particles only, resulting in a molecular concentration of 0.02 as described

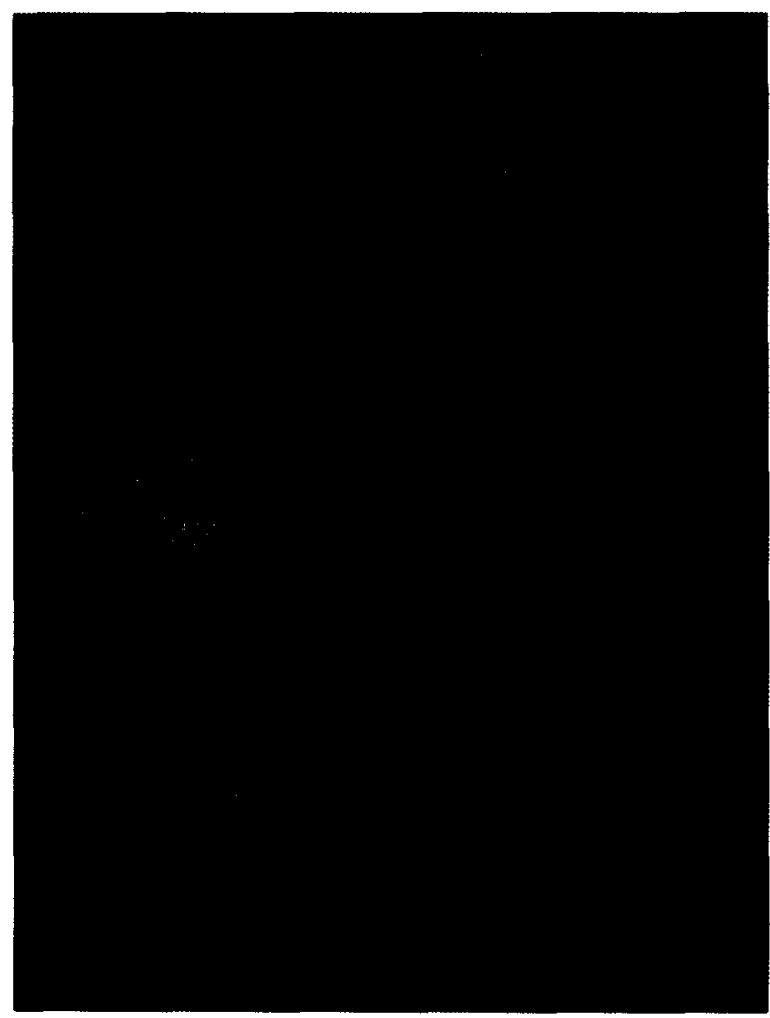

Fig. 10. Snapshot of the system of spherical micelles of H3HT5 surfactants. In addition, some monomeric surfactants are shown. See Fig. 4 for the color scheme.

in Section 5.4. Simultaneously, an oil droplet was created in water by adding $\mathrm{T} 5$ oil molecules to the water. Next, the oil droplet was transferred to the micellar solution. At the initial stagc of onc simulation, the system contained one oil droplet of 426 T5 molecules (see Fig. 13). In a second simulation, the oil droplet consisted initially of 426 T2 and 426 T3 molecules. In addition to the oil droplet, the simulation box contained 471 surfactants incorporated in 23 micelles and 30 additional surfactants not belonging to micelles, and 25096 water molecules. The trajectories of the 31735 particles in the second simulation were followed for 1600000 time steps [35]. The results from this second simulation are briefly discussed below.

As a result of their amphiphilicity, surfactant molecules adsorb at interfaces, and significantly change their behavior $[19,36]$. Fig. 14 shows the amount of adsorbed surfactants at the 


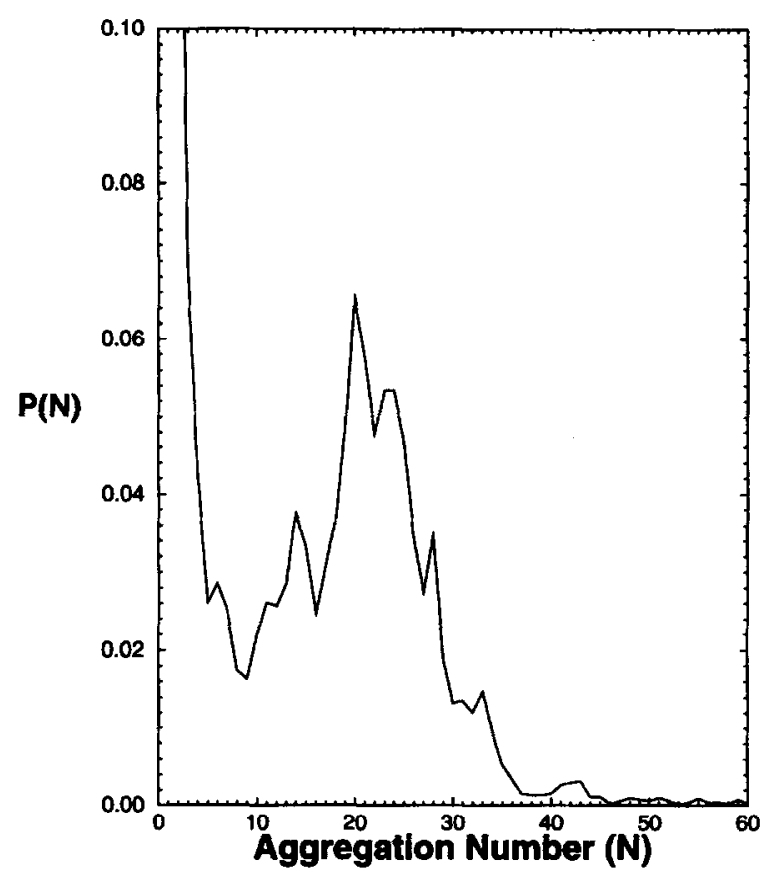

Fig. 11. Micellar size distribution function $P(N)$ where $N$ is the aggregation number for the H3HT5 surfactant. The figure has been obtained by averaging over approximately 200 configurations taken every 4000 th time step.

droplet/water interface versus time. Shortly after the simulation is started, surfactants tend to adsorb on the droplet surface, and as the surface approaches saturation, the amount of adsorbed surfactants per time step decreases in magnitude. Three processes are identified for surfactant adsorption at the oil droplet/water interface. Initially, when the oil droplet surface is void of surfactants, complete micelles adsorb at the interface. At a later stage, surfactant adsorption occurs via exchange of surfactants during micelle-oil droplet collisions, as indicated by the small peaks in the adsorption curve. Furthermore, during all stages, monomeric surfactants adsorb directly at the interface. We expect that the number of peaks in a similar adsorption curve for surfactants that have charged headgroups is much less duc to the long-range repulsion between surfactants adsorbed at the oil droplet and micelles.

One of the significant details of the adsorptiontime curve is the strong decrease in the number of adsorbed surfactants at time step 920000 . This

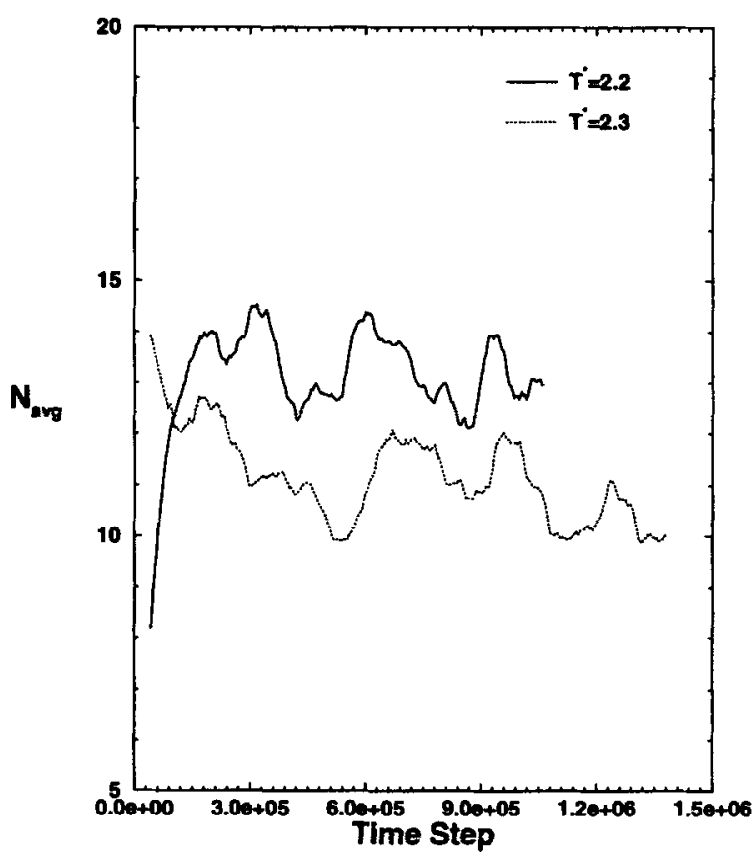

Fig. 12. Average micellar size of H3HT5 as a function of time at two temperatures. The simulation at lower temperature was started from a random placement of the surfactants in water. The last configuration of this simulation was used as an initial configuration for the simulation at higher temperature.

decrease is accompanied by a decrease in the number of oil molecules in the oil droplet (Fig. 14). Close analysis of the data shows that the oil droplet is being broken into two droplets: a large droplet and a small one. This mechanism (mechanism (1)), which was speculated upon by Shaewitz et al. [37], and Carroll [38], is due to low interfacial tension caused by the adsorbed surfactants at the oil droplet surface.

In addition to mechanism (1), oil molecules are transferred from the oil phase to micelles through two other mechanisms. In mechanism (2), oil molecules that leave the oil droplet are trapped by micelles in the immediate vicinity of the droplet. This mechanism is a direct effect of the finite solubility of oil molecules in water. In the presence of micelles, oil molecules in water are being constantly trapped by micelles, creating a chemical potential difference between the oil and water phases, causing a net oil diffusion from the droplet towards water until the correct partition is reached. 


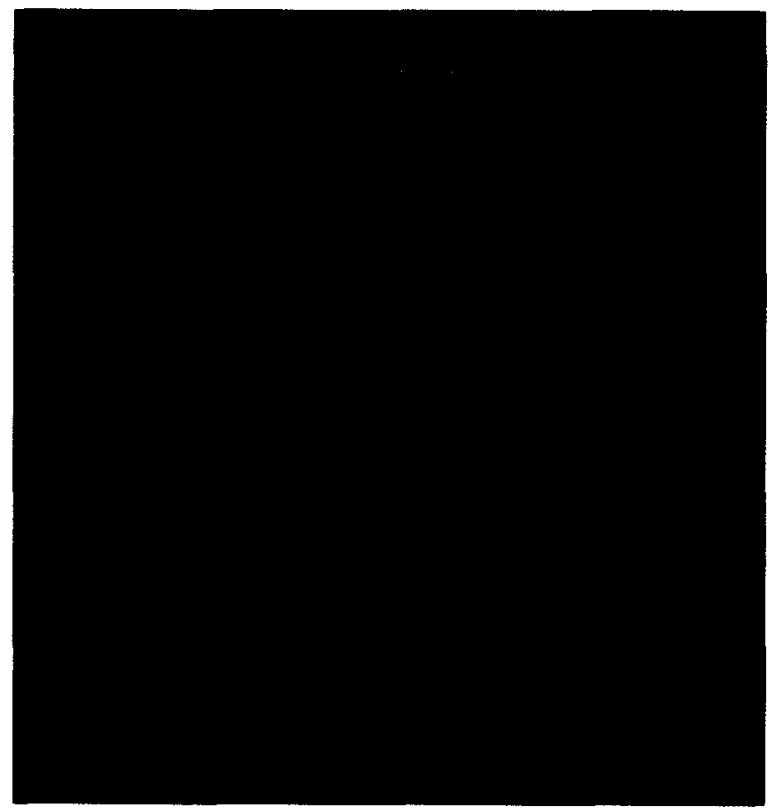

Fig. 13. Snapshot of the initial configuration of oil droplet, micelles and free surfactants for a system of H3HTS surfactants and T5 oil molecules. Surfactant head groups are displayed in yellow, tail segments are shown in red, and oil molecules are drawn in green.

In mechanism (3), oil molecules are transferred through droplet-micelle collisions during which an exchange of surfactants and oil molecules occurs. Collision mechanisms have been suggested for solubilizate exchange between water-in-oil microemulsions and quencher exchange between micelles [39]. In this mechanism, micelles play a carrier role similar to that played by biological cells.

As a direct result of the difference in water solubility, T2 is solubilized more than T3 (Fig. 15). This agrees with experimental data which indicate that molecules with smaller molecular volumes are preferentially solubilized by micellar aggregates, given similar flexibility and polarity of the molecules [40]. While mechanism (2) is clearly favored by short oil molecules, mechanisms (1) and (3) are equally probable for the short and long oil molecules. The ratio of $\mathrm{T} 2$ to $\mathrm{T} 3$ molecules transferred via mechanisms (1) and (3) is equal to $3 / 2$ which is of course related to the difference in size of the two types of oil molecules.

Fig. 16 shows a snapshot of parts of the simula-

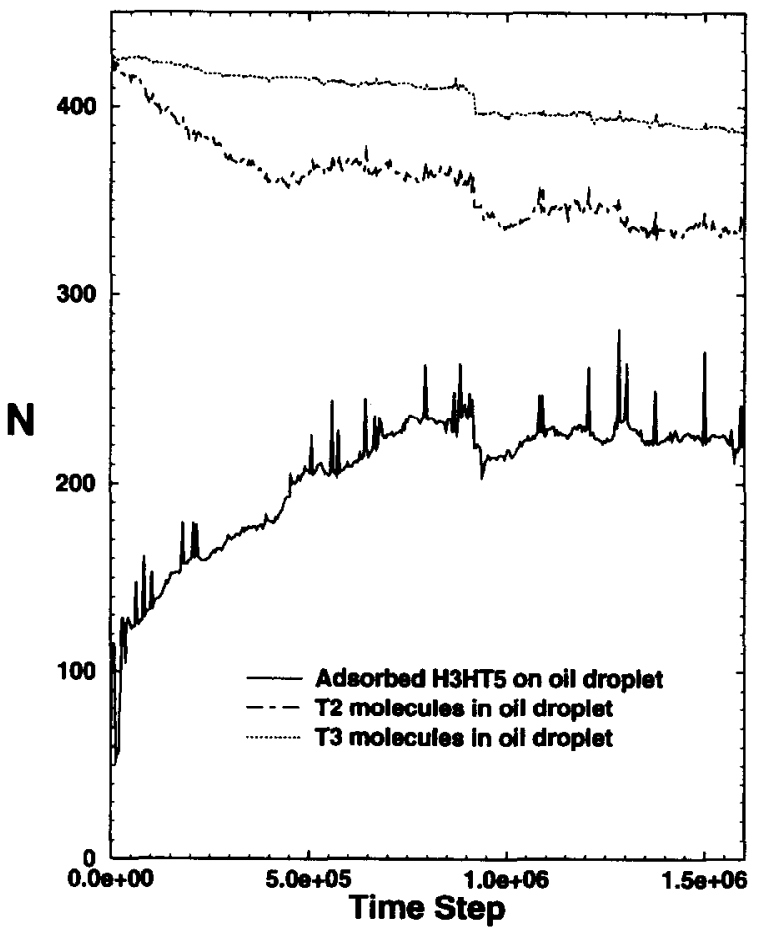

Fig. 14. Number of surfactant molecules adsorbed at the oil droplet/water interface, as well as the number of T2 and T3 molecules which are still in the oil droplet. One of the significant details of the three curves is the presence of a strong decrease in the number of adsorbed surfactants together with a decrease of $\mathrm{T} 2$ and $\mathrm{T} 3$ molecules that are in the oil droplet (time step, 920000).

tion after a significant number of oil molecules have been transferred from the oil droplet to the micelles.

\section{Conclusions}

By applying the technique of molecular dynamics simulation, we have witnessed the capacity of surfactant molecules to self-organize into micellar aggregates with different shapes, as well as the solubilization of oil molecules by such structures. The aggregation of surfactants into micelles near a monolayer causes fluctuations in the density profile of the solvent. These fluctuations are similar to those observed in the density profiles of hard spheres near a wall. The shapes of surfactant aggregates found in our simulations depend 

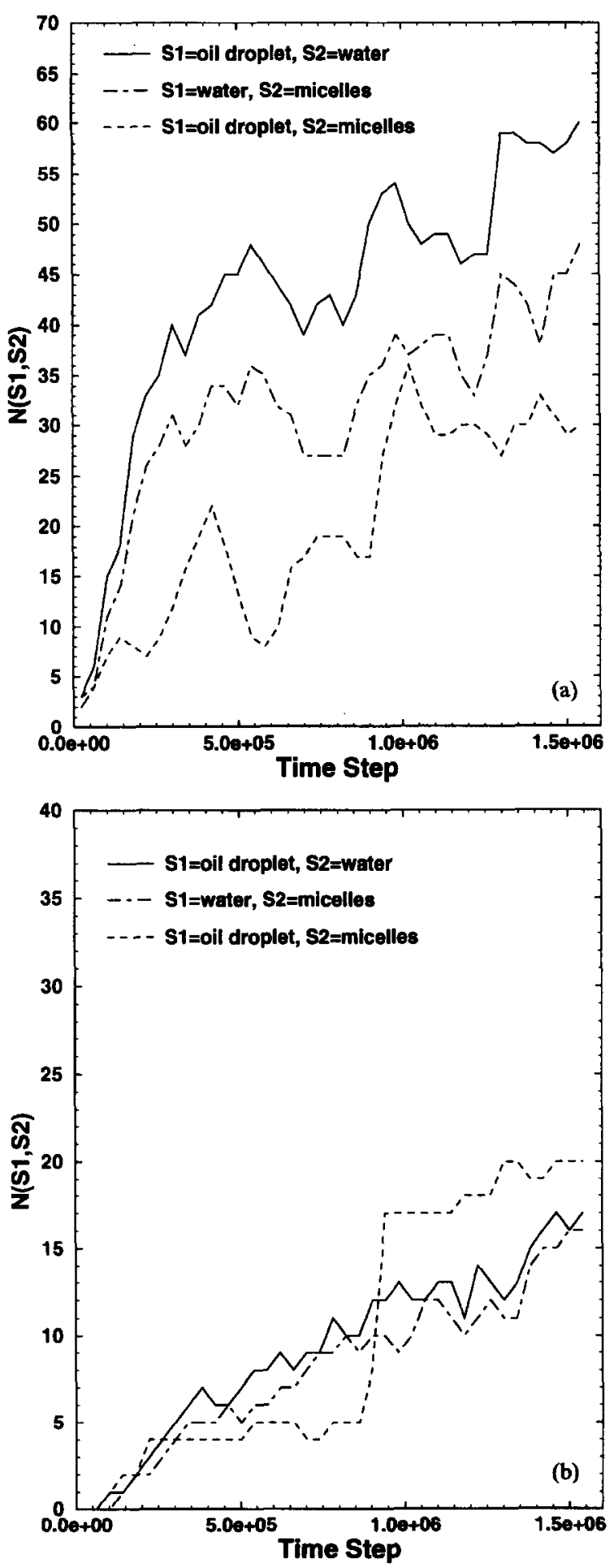

Fig. 15. Cumulative number of (a) T2 and (b) T3 oil molecules that change from state (S1) to state (S2). The three states defined here are the oil droplet, water and micelles.

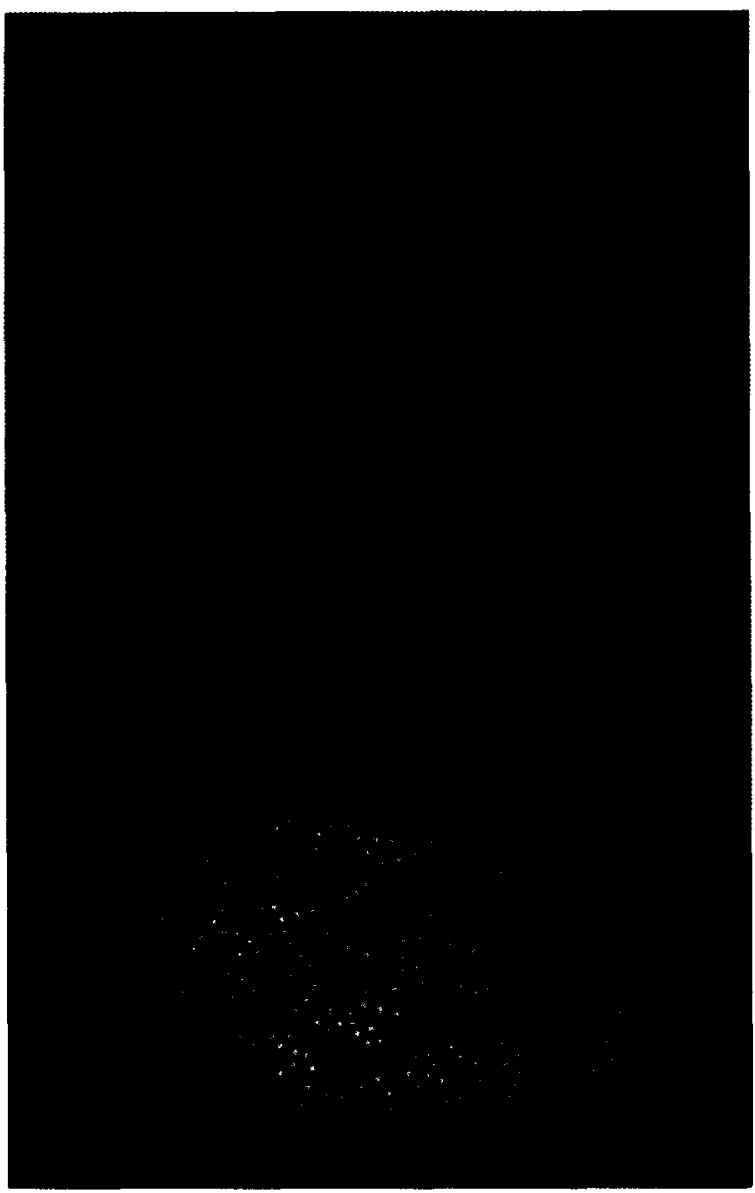

Fig. 16. Snapshot of the final configuration of oil droplet, micelles and free surfactants for a system of H3HT5 surfactants and a mixture of equal number of $\mathrm{T} 2$ and $\mathrm{T} 3$ oil molecules. Surfactant headgroups are displayed in yellow, tail segments are shown in red, $\mathrm{T} 2$ molecules are displayed in green and $\mathrm{T} 3$ molecules in light blue.

to a large extent on the geometry of the surfactant, in accordance with the theory of Israelachvili [27]. The size distribution functions calculated from our equilibrium simulations agree well with the thermodynamic calculations of the aggregate size distribution curve for fairly dilute micellar systems (see Ref. [4] and references cited therein).

Simulations of oil solubilization in aqueous micellar solutions indicate the presence of three mechanisms by which oil molecules are transferred from an oil droplet into micelles. All three mechanisms occur simultaneously, but to different extents, depending upon the molecular details of 
the constituents. In systems in which the oil droplet contains equal numbers of short and long oil molecules, the shorter ones are solubilized to a greater extent because of their higher solubility in the solvent.

It is remarkable that such a simple model shows the self assembly of surfactants into micelles and bilayers. The agreement between simulated micellar shapes and theoretical predictions, and the transfer of oil molecules from an oil-rich phase to a micelle-rich phase are prominent outcomes of our simulation studies. Important to note is that in our model, hydrogen bonds are not present. This suggests that it is possible to form micelles in solvents without hydrogen bonds, in contrast to what is concluded by Beesley et al. [41]. Our observations are consistent with those of Lo Nostro and Chen [33], who observed the selfassembly of micelles in liquids without hydrogen bonds. Our work is not intended to perform quantitative comparisons with experimental data, but is mostly an indication that computer simulations are capable of providing an insight into the structure and dynamics of real systems. This can be expected to become more important in the future as faster computers become available.

\section{References}

[1] M. Allen and D. Tildesley, Computer Simulation of Liquids, Oxford University Press, Oxford, 1987.

[2] M. McBain and E. Hutchinson, Surfactant Solutions: New Methods of Investigation, Solubilization and Related Phenomena, Academic Press, New York, 1955.

[3] P. Elworthy, A. Florence, and C. Macfarlane, Solubilization by Surface-Active Agents and its Applications in Chemistry and the Biological Sciences, Chapman and Hall, London, 1968.

[4] J. Lang and R. Zana, Surfactant Solutions: New Methods of Investigation, in R. Zana (Ed.), Surfactant Science Series, Vol. 22, Marcel Dekker, New York, 1987, Chapter 8, pp. 405-452.

[5] M.T. da Gama and K. Gubbins, Mol. Phys., 59(1986) 227.

[6] B. Smit, P.A.J. Hilbers, K. Esselink, L.A.M. Rupert, N.M. van Os and A.G. Schlijper, Nature, 348 (1990) 624.

[7] L. Verlet, Phys. Rev., 159 (1967) 98.

[8] R. Tarjan, SI $\Lambda$ M (Soc. Ind. $\Lambda$ ppl. Math) J. Comput. 1 (1972) 146.

[9] D. Fincham, Mol. Simulation, 1 (1987) 1.

[10] J. Li, D. Brass, D. Ward and B. Robson, Parallel Comput. 14 (1990) 211.
[11] H.G. Petersen and J.W. Perram, Mol. Phys., 67 (1989) 849.

[12] M. Pinches, D. Tildesley and W. Smith, Mol. Simulation, 6 (1991) 51 .

[13] K. Esselink, B. Smit and P. Hilbers, J. Comput. Phys., 106 (1993) 101.

[14] B. Smit, P.A.J. Hilbers, K. Esselink, L.A.M. Rupert, N.M. van $O s$ and A.G. Schlijper, J. Phys. Chem., 95 (1991) 6361 .

[15] B. Alder and T. Wainwright, J. Chem. Phys., 27 (1957) 1208

[16] D. Frenkel, H. Lekkerkerker and A. Stroobants, Nature, 332 (1988) 822 .

[17] P. Tarazona, U.M.B. Marconi and R. Evans, Mol. Phys., 60 (1987) 573.

[18] A. Nikolov, D.T. Wasan, N.D. Denkov, P.A. Kralchevsky and B. Ivanov, Prog. Colloid Polym. Sci., 82 (1990) 87.

[19] R. Miller and G. Kretzshmar, Adv. Colloid Interface Sci., 37 (1991) 97.

[20] B. Smit, Phys. Rev. A, 37 (1988) 3431.

[21] N.M. van Os, L.A.M. Rupert, B. Smit, P.A.J. Hilbers, K. Esselink, M. Böhmer and L.K. Koopal, Colloids Surfaces, 81 (1993) 217.

[22] S. Karaborni and J. O'Connell, Langmuir, 6 (1990) 905.

[23] S. Karaborni, Langmuir, 9 (1993) 1334.

[24] E. Lee, R. Thomas, J. Penfold and R. Ward, J. Phys. Chem., 93 (1989) 381.

[25] E. Lee, E. Simister, R. Thomas and J. Penfold, Prog. Colloid Polym. Sci., 82 (1990) 99.

[26] J. Lu, E. Simister, R. Thomas and J. Penfold, J. Phys. Chem., 97 (1993) 13907.

[27] J.N. Israelachvili, D.J. Mitchell and B.W. Ninham, J. Chem. Soc., Faraday Trans. 2, 72 (1976) 1525.

[28] R. DeLisi, S. Milioto and R. Triolo, J. Solution Chem., $17(1988) 673$.

[29] E. Roelants and F. de Schryver, Langmuir, 3 (1987) 209.

[30] D.F. Evans and B. Ninham, J. Phys. Chem., 90 (1986) 226.

[31] J.J. Nusselder and J.B. Engberts, J. Org. Chem., 56 (1991) 5522.

[32] S. Karaborni and J. O'Connell, J. Chem. Phys., 92 (1990) 6190.

[33] P. Lo Nostro and S.-H. Chen, J. Phys. Chem., 97 (1993) 6535 .

[34] W. Binana-Limbelé, N. van Os, L. Rupert and R. Zana, J. Colloid Interface Sci., 141 (1991) 157.

[35] S. Karaborni, N. van Os, K. Esselink and P. Hilbers, Langmuir, 9 (1993) 1175.

[36] B.-Y. Zhu and T. Gu, Adv. Colloid Interface Sci. 37 (1991) 1.

[37] J. Shaewitz, A.-C. Chan, E. Cussler and D. Evans, J. Colloid Interface Sci., 84 (1981) 47.

[38] B.J. Carroll, J. Colloid Interface Sci., 79 (1981) 126.

[39] R. Zana, in Surfactant Solutions: New Methods of Investigation, in R. Zana (Ed.), Surfactant Science Series, Vol. 22, Marcel Dekker, New York, 1987, Chapter 5, pp. 241-294.

[40] R. Nagarajan and E. Ruckenstein, Langmuir, 7 (1991) 2934.

[41] A. Beesley, D.F. Evans and R. Laughlin, J. Phys. Chem., 92 (1988) 791. 